\title{
54 PATTERN OF HOSPITAL ADMISSION IN THE FINAL YEAR OF LIFE
}

10.1136/bmjspcare-2011-000053.54

P Lyons, J Verne South West Public Health Observatory, Bristol, UK

This study examines the patterns of hospital care received in the final year of life for the disease groups; cancer, cardiovascular disease (CVD), respiratory and stroke. This will inform policy around End of Life Care pathways and costing models for hospitals.

Our study moves beyond the historical cross-sectional approach, directly linking hospital records to individual death records. The Office of National Statistics (ONS) and Hospital 
Episodes Statistics (HES) linkage provides a longitudinal view of the hospital services received 12 months before death. Using this linked data we have investigated the frequency, length and type of admission (elective, emergency) received.

Our cohort includes individuals that died in England 20042008 and had at least one admission to hospital in the final year of life. We analysed how this varied with underlying cause of death, age, gender, deprivation and Local Authority (LA) of residence.

Our study found that $78 \%$ of all ONS deaths had at least one hospital admission in the year before death; $88 \%$ of cancer deaths and $66 \%$ of CVD deaths had an admission. The average number of admissions a person will have in the final year of life is 3.5 , this varies from Cancer deaths at 5.1 admissions to Stroke deaths with 2.3 admissions. The average number of bed days in the final year of life is 29.7; 36 days for stroke deaths and 18.1 days for CVD. The average length of stay in the final year of life is 8.5 days; 15.7 days for stroke and 5.7 days for Cancer deaths. 